\title{
The effect of electroconvulsive therapy on hematologic inflammatory markers in schizophrenia in association with type of antipsychotic medication
}

\author{
Sinay Önen $\odot$ İbrahim Taymur॰ \\ Department of Pscyhiatry, University of Health Sciences, Bursa Yüksek Ihtisas Training and Research Hospital, Bursa, Turkey
}

\begin{abstract}
Objectives: In recent years there has been an increased interest on the role of inflammation in the pathophysiology of schizophrenia and a search for readily applicable prognostic markers. The impact of electroconvulsive therapy (ECT) on inflammatory function in schizophrenia is still unclear. The aim of this retrospective study is to compare pre- and post-ECT values of red cell distribution width (RDW), mean platelet volume (MPV), mean cell hemoglobin (MCH), mean cell hemoglobin concentration (MCHC), neutrophil-tolymphocyte ratio (NLR) and platelet-to-lymphocyte ratio (PLR) which are considered to be inflammatory markers, and to discuss the findings in context of neuroinflammatory ethiology of schizophrenia.

Methods: Inpatient files were reviewed via complete blood count (CBC), sociodemographic and clinical characteristics (e.g. gender, age, Body Mass Index (BMI), type of psychotropic medication). A total of 58 schizophrenic patients who underwent ECT were were compared in terms of pre- and post-ECT values of RDW, MPV, MCH, MCHC, NLR and PLR in association with type of psychotropic medication.

Results: It was found that MPV, RDW, MCH and MCHC levels significantly decreased after ECT $(p<0.05)$, but no significant difference was found in terms of NLR and PLR $(p>0.05)$. When compared according to the type of psychotropic medication during ECT, MPV and MCHC were decreased after ECT in both typicaland atypical antipsychoticintervention groups $(p<0.05)$. ECT-related inflammatory marker changes were more likely to be associated with atypical antipsychotic medication use during ECT.

Conclusions: Our results indicate that recurrent ECT sessions caused a change in the function of the immune system which might be considered to explain the therapeutic effects of ECT in schizophrenia.
\end{abstract}

Keywords: Electroconvulsive therapy, schizophrenia, inflammatory markers, antipsychotic medication, immune system

chizophrenia is a severe, complex and multifactorial disorder with well-defined symptoms and a lifelong course causing disability. Increasing evidence suggests that immunological and inflammatory mechanisms play important roles in the pathophysiology of schizophrenia. Various immune alterations, such as increased frequency of activated lymphocytes [1] and abnormal levels of inflammatory cytokines $[2,3]$ have been observed in schizophrenic patients. Previous studies have demonstrated an association between elevated plasma inflammatory biomarkers and increased risk of schizophrenia $[4,5]$. It had been suggested that the relationship between the long duration of disease, therapy resistance and high pro-inflammatory cy- 
tokine levels indicatedistinct immune processes might be taking place in different stages of schizophrenia [6]. Previous researches which demonstrated that mean platelet volume, neutrophil-to-lymphocyte ratio, platelet-to-lymphocyte ratio and red blood cell distribution width are correlated with inflammation in chronic diseases (such as cardiovascular diseases, chronic nephropathies, neoplasms, cerebrovascular diseases and autoimmune diseases), and that they may have valuable potential to evaluate the inflammation in these diseases [7-9]; the relationship between these hematologic parameters and immune dysfunction in schizophrenia is still unclear.

The neutrophil-to-lymphocyte ratio (NLR) has been suggested as a new indicator of low-grade inflammation and a predictor of clinical outcomes in neuroimmune disorders $[10,11]$. NLR is previously been studied in major depressive disorder and bipolar disorder and higher NLR levels are detected to be correlated with the severity of MDD which is presented as evidence supporting the inflammatory hypothesis of MDD [12-15]. Cytokine abnormalities and other markers of immune dysfunction were severally identified in schizophrenic patients; but any credible correlation could not be demonstrated between the magnitude of the inflammatory process and the severity of schizophrenia [16]. Previous findings of limited number of studies demonstratedelevated NLR levels in non-obese patients with schizophrenia individuals and a statistically significant positive relationship between PANSS total scores with NLR $[17,18]$. Also, platelet-to-lymphocyte ratio (PLR) is thought to be better than NLR for determining the severity of inflammation [19].

Electroconvulsive therapy (ECT), a procedure which provide faster therapeutic effects, is used for managing the patients with psychiatric disorders, such as major depressive disorder and refractory psychiatric disorders, in acute critical conditions, such as suicidal attempt or severe mania [20,21]. Despite this range of action, the mechanisms by which ECT exerts its beneficial effects remain largely unknown. In a recent study of Kruse et al. [22], the levels of CRP, Interleukin-6 (IL-6), Interleukin-8 (IL-8) and tumor necrosis factor alpha (TNF- $\alpha$ )in 29 patients with treatment resistant depression were compared before ECT treatment and after second session of ECT; and the researchers suggested that acute changes in IL-6 and
CRP had not been related to the alteration of depressive symptom severity over the course of ECT. Recently, Asoğlu et al. [23] investigated the effects of ECT on hematological parameters and reported a significant increase in $\mathrm{MCH}$ and no difference in $\mathrm{MCHC}$, MPV and RDW after repetitive ECT in 25 patients diagnosed with schizophrenia, bipolar disorder and unipolar depression. There is compelling evidence that ECT is closely related to an enhanced innate neuroinflammatory as well as hematogenous immune response. For this reason, it is clear that studies to be done in this area may contribute to explain the mechanisms of ECT's therapeutic effect.

The aim of this retrospective study was to compare hematologic inflammatory markers (NLR, PLR, MPV, $\mathrm{RDW}, \mathrm{MCH}$ and $\mathrm{MCHC}$ ), before and after ECT, which is considered to have mediating roles on the therapeutic effects of ECT; and to discuss in context of neuroinflammatory ethiology of schizophrenia in the light of recent studies.

\section{METHODS}

\section{Study population}

This retrospective study was conducted at Bursa Yüksek İhtisas Training and Research Hospital Psychiatric Inpatient Unit. The study protocol was approved by local Ethics Committee (approval number: 2011-KAEK-25 2018/06-09). Data regarding age, gender, duration of disease, psychiatric diagnosis, indication of ECT, treatment status, type of psychotropic medication before and during hospitalization were collected from the hospital records. A total of 159 patients who had been hospitalized and had been applied ECT between April 2016 and June 2018 were recruited. Ninety-five patients, between 18 and 65 years of age, diagnosed with 'Schizophrenia Spectrum and Other Psychotic Disorders' according to DSM-5 criteria and underwent ECT during hospitalization were included in the study. All inpatient files were reviewed via complete blood count (CBC), sociodemographic and clinical characteristics such as gender, age, body mass index (BMI), duration of disease, previous history of medication, accompanying physical disease (hematological, cardiovascular, liver, rheumatic, etc.). Patients receiving any anti-inflammatory treatment 
(non-steroid anti-inflammatory drug, corticosteroid or other anti-inflammatory drug) and having a history of coronary artery disease/myocardial infarction/heart valve disease, pulmonary disease, rheumatic disease, liver disease, neurological deficit, mental retardation, autism, iron deficiency anemia, bone marrow disease, kidney disease, alcohol and substance use, antiplateletanticoagulant drug use, clozapine use and also morbid obesity, pregnancy and acute infection during hospitalization period were excluded due to the risk of these affecting blood values in association with inflammation. Thus, 16 patients were excluded from the study because of the above comorbid conditions. Twentyone patients, whose post-ECT CBC results had not been available in the files, were also excluded from the study.

\section{Data collection}

Thus, a total of 58 schizophrenic patients (27 females and 31 males) were enrolled in the study. All patients had been initiated on psychotropic medication on the admission, which had not been altered during ECT procedure. Antipsychotic prescription data before hospitalization and during ECT intervention were also noted; antipsychotic prescription was further coded as typical (TAP), atypical (AAP) and combined typicalatypical (CAP) to examine for differences in hematologic values.

The patients underwent ECT procedure with a MECTA spECTrum 5000 ECT device, 3 times a week. The effective duration of seizure was determined to be at least 20 seconds. ECT was administered in the morning after an overnight fasting. Moreover, intravenous propofol and rocuronium bromide were administered to all patients in order to induce anesthesia and muscle relaxation. Blood samples being taken the day before the first session of ECT (pre-ECT) and the day after the $7^{\text {th }}$ session of ECT (post-ECT) were extracted from the laboratory files to exclude bias due to different number of ECT sessions. The following parameters were reviewed from the inpatient laboratory files for all the subjects: Red cell distribution width (RDW), mean platelet volume (MPV), mean cell hemoglobin (MCH) and mean cell hemoglobin concentration (MCHC). The neutrophilto-lymphocyte ratio (NLR) was calculated by dividing the absolute neutrophil count by the absolute lymphocyte count. The platelet-to-lymphocyte ratio
(PLR) was calculated by dividing the absolute platelet count by the absolute lymphocyte count.

\section{Statistical Analysis}

Statistical Package for Social Sciences (SPSS v22, Chicago, IL, U.S.A.) programme for Windows was used. Descriptive parameters are expressed as mean, standard deviation or percentage. After performing Kolmogorov-Smirnov test the distribution normality of the quantitative variables, independent t-test was used for the normally distributed variables and Kruskal-Wallis and Wilcoxon test was used for the non-normally distributed variables. The significance level was set as $p<0.05$.

\section{RESULTS}

A total of 58 schizophrenic patients ( 27 females and 31 males) were evaluated in this study. The mean age of the study group was $39.43 \pm 10.43$ years. The mean duration of disease was $12.98 \pm 8.51$ years. Of the 58 patients, $44.8 \%$ of them $(n=26)$ had been

$\begin{aligned} & \text { Table 1. Demographic characteristics of the } \\
& \text { schizophrenic patients }(\mathbf{n = 5 8})\end{aligned}$
\begin{tabular}{lc}
\hline Characteristics & Data \\
\hline Age (years) & $39.43 \pm 10.43$ \\
Duration of disease (years) & $12.98 \pm 8.51$ \\
BMI $\left(\mathrm{kg} / \mathrm{m}^{2}\right)$ & $26.96 \pm 3.73$ \\
Gender & \\
\multicolumn{1}{l}{ Female } & $27(\% 46.6)$ \\
$\quad$ Male & $31(\% 53.4)$
\end{tabular}

Regular psychotropic medication before hospitalization

\begin{tabular}{|cc|}
\hline Yes & $26(44.8 \%)$ \\
\hline No & $32(55.2 \%)$ \\
\hline Indication of ECT & \\
\hline Treatment resistance & $42(72.4 \%)$ \\
\hline Refusal of oral intake & $6(10.3 \%)$ \\
\hline Severe agitation & $4(6.9 \%)$ \\
\hline Suicidal ideation & $3(5.2 \%)$ \\
\hline Catatonic symptoms & $3(5.2 \%)$ \\
\hline
\end{tabular}

Data are shown as as mean \pm standart deviation or $\mathrm{n}(\%)$. $\mathrm{BMI}=$ Body mass index, $\mathrm{ECT}=$ Electroconvulsive therapy 
Table 2. Comparison of pre-ECT and post-ECT of hematological inflammatory markers

\begin{tabular}{llcc}
\hline & & Mean \pm SD & p value \\
\hline PLR & Pre-ECT & $116.40 \pm 47.36$ & 0.421 \\
\hline \multirow{2}{*}{ NLR } & Post-ECT & $119.46 \pm 41.54$ & \\
& Pre-ECT & $2.50 \pm 1.11$ & 0.769 \\
MPV (fL) & Post-ECT & $2.72 \pm 1.70$ & $0.000^{* *}$ \\
& Pre-ECT & $8.51 \pm 0.87$ & $0.004^{*}$ \\
RDW (\%) & Post-ECT & $8.04 \pm 0.81$ & \\
& Pre-ECT & $13.40 \pm 1.43$ & $0.031^{*}$ \\
MCH (pg) & Post-ECT & $13.01 \pm 1.10$ & \\
& Pre-ECT & $28.79 \pm 2.07$ & $0.003^{*}$ \\
\hline \multirow{2}{*}{ MCHC (g/dL) } & Post-ECT & $28.48 \pm 1.69$ & \\
& Pre-ECT & $33.28 \pm 0.74$ & $33.03 \pm 0.82$ \\
\hline
\end{tabular}

Wilcoxon Signed Rank Test was used. ECT $=$ Electroconvulsive therapy, Pre-ECT $=$ Before electroconvulsive therapy, Post-ECT $=$ After electroconvulsive therapy, PLR $=$ Platelet-to-lymphocyte ratio, NLR $=$ Neutrophil-tolymphocyte ratio, MPV $=$ Mean platelet volume, $\mathrm{RDW}=$ Red cell distribution width, $\mathrm{MCH}=$ Mean cell hemoglobin, $\mathrm{MCHC}=$ Mean cell hemoglobin concentration, SD: standard deviation

receiving regular psychotropic medication before hospitalization. Of these 26 patients, 10 were having typical (TAP), 15 were having atypical (AAP), 9 were having combined typical and atypical antipsychotic (CAP) medication. None of the patients were using clozapine before hospitalization. According to ECT indications; $72.4 \%(\mathrm{n}=42)$ of the patients were underwent ECT for treatment resistance; $10.3 \%(\mathrm{n}=$ 6) for refusal of oral intake; $6.9 \%(n=4)$ for severe agitation, $5.2 \%(n=3)$ for suicidal ideation and $5.2 \%$ $(\mathrm{n}=3)$ for catatonic symptoms. The mean BMI of the study group was $26.96 \pm 3.73 \mathrm{~kg} / \mathrm{m} 2$ on the admission. Socio-demographic and clinical characteristics of the patient group are given in Table 1. According to the comparison of inflammatory markers; there were statistically significant difference between pre-ECT and post-ECT measures of MPV, RDW, MCH and $\operatorname{MCHC}(p<0.001, p=0.004, p=0.031$ and $p=0.003$, respectively); but no significant difference was found in terms of NLR and PLR $(p=0.769$ and $p=0.421$,

Table 3. Inflammatory markers according to type of psychotropic medication before ECT

\begin{tabular}{|c|c|c|c|c|}
\hline & $\begin{array}{c}\text { TAP } \\
(n=10)\end{array}$ & $\begin{array}{c}\text { AAP } \\
(n=15)\end{array}$ & $\begin{array}{c}\text { CAP } \\
(n=9)\end{array}$ & p value \\
\hline PLR & $113.15 \pm 42.75$ & $126.59 \pm 58.2$ & $112.47 \pm 20.33$ & 0.83 \\
\hline NLR & $2.16 \pm 0.74$ & $2.78 \pm 1.41$ & $2.56 \pm 0.77$ & 0.47 \\
\hline MPV (fL) & $7.54 \pm 0.58$ & $9.30 \pm 0.49$ & $8.16 \pm 0.48$ & $0.000^{* *}$ \\
\hline RDW (\%) & $13.58 \pm 1.58$ & $13.62 \pm 1.22$ & $13.77 \pm 2.01$ & 0.91 \\
\hline $\mathrm{MCH}(\mathrm{pg})$ & $28.99 \pm 1.59$ & $28.42 \pm 3.08$ & $27.82 \pm 1.88$ & 0.38 \\
\hline $\mathrm{MCHC}(\mathrm{g} / \mathrm{dL})$ & $33.23 \pm 0.47$ & $33.02 \pm 0.89$ & $33.23 \pm 0.84$ & 0.80 \\
\hline
\end{tabular}


Table 4. Inflammatory markers before and after ECT procedure according to type of psychotropic medication during hospitalization

\begin{tabular}{llcccc}
\hline & & TAP & p value & AAP & p value \\
\hline PLR & Pre-ECT & $108.69 \pm 36.78$ & 0.411 & $124.66 \pm 56.08$ & 0.716 \\
& Post-ECT & $114.47 \pm 37.47$ & & $124.81 \pm 45.57$ & \\
NLR & Pre-ECT & $2.37 \pm 0.91$ & 0.400 & $2.62 \pm 1.30$ & 0.665 \\
& Post-ECT & $2.88 \pm 2.01$ & & $2.55 \pm 1.30$ & \\
MPV (fL) & Pre-ECT & $8.26 \pm 0.94$ & $<\mathbf{0 . 0 0 1}$ & $8.77 \pm 0.70$ & $<\mathbf{0 . 0 0 1}$ \\
& Post-ECT & $7.96 \pm 0.96$ & & $8.12 \pm 0.62$ & \\
\hline \multirow{2}{*}{ RDW (\%) } & Pre-ECT & $13.43 \pm 1.37$ & 0.120 & $13.37 \pm 1.52$ & 0.114 \\
& Post-ECT & $12.94 \pm 0.90$ & & $13.10 \pm 1.30$ & \\
MCH (pg) & Pre-ECT & $28.74 \pm 2.43$ & 0.420 & $28.84 \pm 1.64$ & 0.367 \\
& Post-ECT & $28.32 \pm 1.81$ & & $28.66 \pm 1.57$ & \\
\hline \multirow{2}{*}{ MCHC (g/dL) } & Pre-ECT & $33.34 \pm 0.85$ & $\mathbf{0 . 0 3 1}$ & $33.23 \pm 0.62$ & $\mathbf{0 . 0 3 2}$ \\
& Post-ECT & $33.02 \pm 0.89$ & & $33.04 \pm 0.75$ & \\
\hline
\end{tabular}

Data are shown as mean \pm standart deviation. Wilcoxon Signed Rank Test was used. TAP = typical antipsychotic, $\mathrm{AAP}=$ atypical antipsychotic, ECT = electroconvulsive therapy, Pre-ECT $=$ Before electroconvulsive therapy, Post-ECT = After electroconvulsive therapy, PLR = platelet-to-lymphocyte ratio, NLR= neutrophil-tolymphocyte ratio, $\mathrm{MPV}=$ mean platelet volume, $\mathrm{RDW}=$ red cell distribution width, $\mathrm{MCH}=$ mean cell hemoglobin, $\mathrm{MCHC}=$ mean cell hemoglobin concentration

respectively). It was found that MPV, RDW, MCH and MCHC levels significantly decreased after 7 sessions of ECT (Table 2).

NLR, PLR, MPV, RDW, MCH and MCHC values were compared according to the type of psychotropic medication before hospitalization. Only MPV was found to be significantly different between three groups (TAP, AAP and CAP) $(p<0.001)$. The mean MPV was $7.54 \pm 0.58$ in patients using TAP $(\mathrm{n}=10)$; $9.3 \pm 0.49$ in patients using AAP $(\mathrm{n}=15)$ and $8.16 \pm$ 0.48 in patients using CAP $(n=9)$ (Table 3$)$.

The study parameters were also compared according to the type of psychotropic medication during ECT intervention. Of the total 58 patients, 30 of them was initiated on TAP and 25 of them was initiated on AAP (except for clozapine) on the admission; which had not been altered during ECT procedure. None of the patients underwent combined antipsychotic medication during ECT. There were statistically significant difference between pre-ECT and post-ECT values of MPV and MCHC in the both TAP ( $p<0.001$ and $p=0.031$, respectively) and AAP groups ( $p<0.001$ and $p=0.032$, respectively). Both MPV and MCHC were decreased after ECT in both medication groups. No significant difference was found in terms of NLR, PLR, RDW and MCH values in both groups (Table 4).

Since MPV changes before and after ECT were significant in both atypical and typical antipsychotic use during ECT and MPV was normally distributed, Independent t-test was done to examine which MPV change is more significant between the different

Table 5. Change in MPV according to type of psychotropic medication during ECT procedure

\begin{tabular}{|c|c|c|c|c|c|c|c|}
\hline \multirow[t]{2}{*}{ MPV change } & \multirow[t]{2}{*}{ Mean \pm SD } & \multirow[t]{2}{*}{ Mean difference } & \multirow[t]{2}{*}{ p value } & \multirow[t]{2}{*}{$d f$} & \multirow[t]{2}{*}{$t$} & \multicolumn{2}{|c|}{$95 \% \mathrm{CI}$} \\
\hline & & & & & & Lower & Upper \\
\hline TAP (\%) & $3.57 \pm 4.68$ & -3.763 & 0.004 & 56 & -2.998 & -6.278 & -1.248 \\
\hline AAP $(\%)$ & $7.33 \pm 4.87$ & & & & & & \\
\hline
\end{tabular}

Independent t-test was used. TAP $=$ typical antipsychotic, AAP $=$ atypical antipsychotic, MPV $=$ mean platelet volume, $\mathrm{ECT}=$ electroconvulsive therapy, $\mathrm{SD}=$ standard deviation, $\mathrm{CI}=$ confident interval, 
antipsychotic types. Mean MPV change was $3.57 \pm$ $4.68 \%$ in TAP group and $7.33 \pm 4.87 \%$ in AAP group $(\mathrm{t}=-2.998, \mathrm{df}=56, p=0.004)($ Table 5).

\section{DISCUSSION}

To the best of our knowledge, this is the first study examining the effects of ECT on hematologic inflammatory markers as NLR, PLR, MPV, RDW, $\mathrm{MCH}$ and $\mathrm{MCHC}$ with regard to its anti-inflammatory effects in schizophrenia. The present study mainly suggested that MPV, RDW, MCH and MCHC were decreased but NLR and PLR did not differ after ECT. Also, it is proposed that the mean MPV values differ in schizophrenicpatients according to the type of medication. In contrast, MPV and MCHC were found to be differed after ECT independently of type of antipsychotic medication.

Animal studies have demonstrated that electroconvulsive seizures induce several changes in neurotrophin and immune signaling, both in the central nervous system (CNS)and in peripheral tissues. Fluitman et al. [24] showed that acute ECT (15-30 min.after the electrostimulus) induces a leukocytosis in MDD patients, driven by significantincreases in absolute numbers of granulocytes, monocytes and natural killer cells. A similar leukocyte pattern of polymorphonuclear leukocytosis and relative lymphopeniawas observed 2 hour after a single ECT in a previous study[25].

The abnormalities of the immune system seen in schizophrenia and related psychosis are varied and overlapping, and involve many immune components [26]. Thus, many current studies have inadequately accounted for confounding factors such as body mass, smoking, and medication to fully understand the role of inflammation and immunity in neuropsychiatric disorders. In this study we also evaluated some factors such as duration of disease and type of psychotrop medication before and during ECT intervention to exclude partially their confounding effects.

It has been reported that obesity is associated with a low-grade inflammatory process in the white adipose tissue (WAT) [27, 28]. Increased mass of adipose tissue simultaneously activates the inflammatory process in WAT itself, in the liver and in immune cells [29]. In our study the mean BMI of the schizophrenia group was less than obesity range $\left(<30 \mathrm{~kg} / \mathrm{m}^{2}\right)$ on the admission and thus, the confounding effect of obesity on inflammatory changes was ensured.

A meta-analysis of 26 cross-sectional or longitudinal studies demonstrated that increased levels of C-reactive protein (CRP) in schizophrenic patients was parallel to the severity of positive symptoms but not associated with the initiation of antipsychotic medication; and the researchers suggested that CRP might be a marker of systemic low-grade inflammation [30]. The NLR can be derived from the white blood cell count, and is inexpensive, replicable and also it has been found to be significantly correlated with CRP [31]. It has been suggested as a new indicator of low-grade inflammation and a predictor of clinical outcomes inneuroimmune disorders $[10,11]$. Previously, NLR is found to be higher in patients with MDD, bipolar disorder and schizophrenia[14, 17, 32-34]. Özdin et al. [14] have shown that PLR values like NLR are higher in schizophrenia patients than healthy controls and bipolar disorder patients, and authors have suggested that the sefindings support the imflammatory hypothesis of schizophrenia and bipolar disorder. However, to date one study has been encountered in the literature investigating the effect of ECT on NLR and in this study it is noted that no significant difference was found in NLR after repeated ECT treatments in 61 patients with schizophrenia, bipolar disorder and depression [35]. The authors argued that the use of psychotropic drugs has not been evaluated and the few number of patients in the subgroups according to the diagnosis may have affected the results of the study. Unlike previous studies, the type of antipsychotic treatment before hospitalization and during ECT was also assessed in our study. Our results showed that antipsychotic treatment both before and during ECT treatment did not cause any significant changes in NLR after ECT intervention. Some previous data suggest that cytokine levels, including IL-6, are associated with severity and duration of schizophrenia and antipsychotic therapy [26, 36, 37].As the vast majority of patients indication of ECT were treatment resistance and the mean duration of disease was $12.98 \pm 8.51$ years; so the difficulty of suppressing the inflammatory response by somatic therapies in patients with long duration of schizophrenia and treatment-resistance might be the 
reason why NLR and PLR did not altered with ECT in our study.

Wysokinski and Szczepocka [38] examined 1243 patients with schizophrenia and showed a significantly lower PLT count and significantly higher MPV value compared with bipolar disorder and unipolar depressive patients. In a study of Lee et al. [39], which was carried out with 100 patients who were initiated on clozapine, the researchers demonstrated higher MPV in patients with schizophrenia, which was unaltered after 1 year of clozapine treatment. Except for the recent study of Asoğlu et al. [23], that could not found any significant difference between pre- and post-ECT MPV values in schizophrenic patients, there were no studies evaluating MPV levels in patients underwent ECT in the literature. Our results demonstrated that mean MPV was higher in patients using atypical antipsychotic medication than typical antipsychotic medication on the admission. When compared according to the type of antipsychotic drug used during ECT, MPV was found to be decreased after ECT in patients receiving both typical and atypical antipsychotic treatment in our study. Furthermore, univariate analysis showed that MPV was reduced by $7.33 \%$ in patients underwent atypical antipsychotic medication, while it was reduced by $3.57 \%$ in patients underwent typical antipsychotic medication during ECT. Considering the fact that the mean MPV values were higher in patients using atypicalantipsychotics before hospitalization and more MPV reduction was observed in patient sunder went atypical antipsychotic medication after ECT, it can be concluded that ECT-related inflammatory marker changes were more likely to be associated with atypical antipsychotic medication use. We suppose that this is one of the most important finding that differ our study from previous studies.

The relationship between inflammation and anemia in some disease is well described and it has been shown that inflammation enforce both direct and indirect effects on erythropoiesis [40]. Ayyildiz et al. [41] found higher RDW in 518 schizophrenic patients than healthy controls in their retrospective study and suggested that RDW might be a helpful diagnostic and prognostic marker of schizophrenia with potential utility in risk estimation and treatment monitoring. Another study with a smaller sample comparing MPV, RDW and vitamin B12 levels in schizophrenic patients and healthy controls claimed that higher RDW and MPV is associated with the inflammatory hypothesis of schizophrenia [42]. According to our study results, like MPV, RDW is also significantly decreased after ECT.

Huang and $\mathrm{Hu}$ [43] found decreased levels of $\mathrm{MCHC}$ in acute myocardial infarction (AMI) and speculated that similar to RDW, MCHC can be regarded asan inflammatory marker and thus can affect the prognosis after AMI. Ayyildiz et al. [41] determined higher $\mathrm{MCH}$ and lower $\mathrm{MCHC}$ in schizophrenic patients compared to healthy controls. When the literature was reviewed, only one study could be found assessing hematologic differences in schizophrenic patients after ECT. In the same study Asoglu et al. [23] reported a significant increase in $\mathrm{MCH}$ and no difference in MCHC, MPV and RDW after repetitive ECT in 25 patients diagnosed with schizophrenia, bipolar disorder and unipolar depression. In our study we determined a significant decrease in both $\mathrm{MCHC}$ and $\mathrm{MCH}$ after ECT, which are inconsistent with the previous relatively smaller sample sized study findings. It can be asserted that our findings are more representative due to the higher number of schizophrenic patients included. There was no difference in $\mathrm{MCH}$ and MCHC according to the type of medication before hospitalization. But when all patients were grouped due to their type of medication during ECT, only MCHC showed significant change between pre- and post-ECT measures. It can be speculated that $\mathrm{MCHC}$ might be considered as a better indicator of inflammatory response than $\mathrm{MCH}$ according to our results.

For a long time it has been known that antipsychotic drugs have immunmodulatory effects [44]. It has been asserted that antipsychotic agents might exert varying effects on immune system mediated by their effect on weight gain and increased adiposity [44]. Previous studies showed that both atypical and typical antipsychotic agents stimulate the production of anti-inflammatory cytokines and suppress the production of pro-inflammatory cytokines [45]. Antipsychotic-naive first episode psychosis and acute psychotic relapse are also found to be associated with increased serum proinflammatory cytokine levels and decreased anti-inflammatory cytokine levels, which are returned 
to previous serum concentrations after remission of symptoms with antipsychotic treatment [36, 46]. Semiz et al. [47] ascertained that MPV levels are influenced by both atypical antipsychotics and schizophrenia itself. According to our findings, only MPV and MCHC values were decreased after ECT in both groups of schizophrenic patients having TAP or AAP treatment, but the mean MPV change was more in AAP group. Thus, it can be speculated that ECT may have anti-inflammatory effects by decreasing MPV levels and this effect is more prominent in patients having atypical antipsychotic treatment.

Propofol is one of the most widely used, safe intravenous anesthetic agent with minimal side effects [48] which has been shown to have anti-inflammatory effects by inhibiting neutrophil functions and the release of pro-inflammatory cytokines $[49,50]$. In the literature, no evidence could be found investigating the impact of rocuronium bromide on inflammatory markers. Although there are some data on the shortterm anti-inflammatory effects of propofol [51], we consider that the evaluation of hematologic inflammatory parameters a day after the last session of ECT anesthesia provide support toexclude the confounding anti-inflammatory effects of propofol on our hematological variables.

Our study has some strengths but as well as a number of limitations. The most important limitation of this study was the retrospective file screening design and therefore the relation with the clinical response could not be evaluated. CRP was not routinely evaluated before ECT; so, we could not be able to assess CRP levels due to the retrospective design of the study. The fact that chlorpromazine equivalent antipsychotic drug doses were not specified is another limitation of our study. In addition, pre-ECT hematologic parameters of patients were not compared with a healthy control group. Another limitation is that accompanying depressive symptoms, which might be an important potential confounder on either ECT and inflammation, and the severity of psychotic symptoms before and after ECT was not measured with clinical evaluation scales.

Although there are small sample studies evaluating some inflammatory markers in patients with major depression, bipolar disorder and schizophrenia who had undergone ECT [22, 23], our study is the first in the literature that extensively handle the effect of ECT on hematologic inflammatory markers in patients with schizophrenia: (1) The evaluation of a wide range of indications for ECT, (2) the fact that only schizophrenic patients were included in this study, (3) the assesment of type of psychotropic drug use before and during ECT, (4) exclusion of risk factors that affect hematologic parameters (like clozapine use and obesity), and (5) the fact that preand post-ECT blood samples have been collected within certain time, are the strengths of this study. We suggest that the larger sample size, evaluation of a wider hematologic profile and research of a longerterm effects of ECT on these easily applicable hematologic parameters makes our study different from previous studies and provide a distinct perspective on the immunological effects of ECT.

\section{CONCLUSION}

ECT is one of the oldest and most effective biological treatment methods in psychiatry which unfortunately lose its popularity from time to time. In the literature there is limited number of studies that examine the effect of ECT on the immune system. Previous researches assessing the effects of ECT on inflammatory response are mainly had focused on changes in anti-inflammatory and pro-inflammatory cytokine levels, but unfortunately cytokine levels do not appear to be cost-effective markers in everyday practice. It is obvious that inflammatory markers that are easier and faster to implement, compatible with clinical features and predicting prognostic factors are needed.

In conclusion, NLR, PLR, RDW, MPV, MCH and $\mathrm{MCHC}$, which are easy to apply and inexpensive inflammatory markers, were evaluated before and after ECT in the present study. Both MPV and RDW levels in schizophrenia patients were found to be decreased after 7 sessions of ECT treatment. According to our results, it can be affirmed that recurrent ECT sessions caused a change in the function of the immune system. This finding supports the hypothesis of alteration in the immune system, which is asserted to explain the mechanism of action of ECT. Because of the fact that all of 58 patients were receiving psychotropic medication during ECT, it seems to be difficult to claim that the change in 
hematologic parameters are due to ECT intervention alone. In order to obtain more robust evidence about the effect of ECT on hematologic inflammatory markers further researches (1) carried out with patients who had the first psychotic episode and did not receive additional psychotropic medication, (2) excluded confounding factors such as smoking and obesity besides drug use, (3) evaluated other immunological markers, like cytokines, with inflammatory markers and (4) with wider patient samples are required to be conducted. There is a need of scope for new research in schizophrenia over the coming years, encompassing immune, genetic, microbiological, and other biomarkers.

\section{Conflict of interest}

The authors disclosed no conflict of interest during the preparation or publication of this manuscript.

\section{Financing}

The authors disclosed that they did not receive any grant during conduction or writing of this study.

\section{REFERENCES}

1. Nikkilä HV, Müller K, Ahokas A, Rimón R, Andersson LC. Increased frequency of activated lymphocytes in the cerebrospinal fluid of patients with acute schizophrenia. Schizophr Res 2001;49:99-105.

2. Potvin S, Stip E, Sepehry AA, Gendron A, Bah R, Kouassi E, et al. Inflammatory cytokine alterations in schizophrenia: a systematic quantitative review. Biol Psychiatry 2008;63:801-8.

3. Müller N. The role of anti-inflammatory treatment in psychiatric disorders. Psychiatr Danub 2013;25:292-8.

4. Benros M, Nielsen P, Nordentoft M, Eaton W, Dalton S, Mortensen P. Autoimmune diseases and severe infections as risk factors for schizophrenia: a 30-year population-based register study. Am J Psychiatry 2011;168:1303-10.

5. Wium-Andersen MK, Orsted DD, Nordestgaard BG. Elevated $\mathrm{C}$-reactive protein associated with late- and very-late- onset schizophrenia in the general population: a prospective study. Schizophrenia Bull 2014;40:1117-27.

6. Müller N, Schwarz MJ. Immune system and schizophrenia. Curr Immunol Rev 2010;6:213-20.

7. Kuyumcu ME, Yesil Y, Ozturk ZA, Kizilarslanoglu C, Etgül $\mathrm{S}$, Halil M, et al. The evaluation of neutrophil-lymphocyte ratio in Alzheimer's disease. Dement Geriatr Cogn Disord 2012;34:6974.

8. Balta S, Demirkol S, Kucuk U. The platelet lymphocyte ratio may be useful inflammatory indicator in clinical practice. Hemodial Int 2013;17:668-9.
9. Kopuz A, Turan V, Ozcan A, Kopuz Y, Toz E, Kurt S. A novel marker for the assessment of the treatment result in pelvic inflammatory disease. Minerva Ginecol 2016;68:117-23.

10. Akıl E, Bulut A, Kaplan I, Özdemir HH, Arslan D, Aluçlu MU. The increase of carcinoembryonic antigen (CEA), highsensitivity C-reactive protein, and neutrophil/lymphocyte ratio in Parkinson's disease. Neurol Sci 2015;36:423-8.

11. Rembach A, Watt AD, Wilson WJ, Rainey-Smith S, Ellis KA, Rowe CC, et al. An increased neutrophil-lymphocyte ratio in Alzheimer's disease is a function of age and is weakly correlated with neocortical amyloid accumulation. J Neuroimmunol 2014;273:65-71.

12. Kalelioglu T, Akkus M, Karamustafalioglu N, Genc A, Genc ES, Cansiz A, et al. Neutrophil-lymphocyte and plateletlymphocyte ratios as inflammation markers for bipolar disorder. Psychiatry Res 2015;228:925-7.

13. Gündüz N, Timur Ö, Erzincan E, Turgut C, Turan H, Akbey ZY. Evaluation of mean platelet volume, neutrophil/lymphocyte ratio, platelet/lymphocyte ratio, and red cell distribution width in patients with diagnosis of major depressive disorder. Med Med J 2017;32:230-7.

14. Özdin S, Sarisoy G, Böke Ö. A comparison of the neutrophillymphocyte, platelet-lymphocyte and monocyte-lymphocyte ratios in schizophrenia and bipolar disorder patients - a retrospective file review. Nord J Psychiatry 2017;71:509-12.

15. Aykut DS, Arslan FC, Karagüzel EÖ, Aral G, Karakullukçu $\mathrm{S}$. The relationship between neutrophil-lymphocyte, plateletlymphocyte ratio and cognitive functions in bipolar disorder. Nord J Psychiatry 2018;72:119-23.

16. Manu P, Correll CU, Wampers M, Mitchell AJ, Probst M, Vancampfort D, et al. Markers of inflammation in schizophrenia: association vs. causation. World Psychiatry 2014;13:189-92.

17. Semiz M, Yildirim O, Canan F, Demir S, Hasbek E, Tuman TC, et al. Elevated neutrophil/lymphocyte ratio in patients with schizophrenia. Psychiatr Danub 2014;26:220-5.

18. Kulaksizoglu B, Kulaksizoglu S. Relationship between neutrophil/lymphocyte ratio with oxidative stress and psychopathology in patients with schizophrenia. Neuropsychiatr Dis Treat 2016;12:1999-2005.

19. Turkmen K, Erdur FM, Ozcicek F, Ozcicek A, Akbas EM, Ozbicer A, et al. Platelet-to-lymphocyte ratio better predicts inflammation than neutrophil-to-lymphocyte ratio in end-stage renal disease patients. Hemodial Int 2013;17:391-6.

20. Keshtkar M, Ghanizadeh A, Firoozabadi A. Repetitive transcranial magnetic stimulation versus electroconvulsive therapy for the treatment of major depressive disorder, a randomized controlled clinical trial. J ECT 2011;27:310-4.

21. Jahangard, L, Haghighi M, Bigdelou G, Bajoghli H, Brand S. Comparing efficacy of ECT with and without concurrent sodium valproate therapy in manic patients. J ECT 2012;28:11823.

22. Kruse JL, Congdon E, Olmstead R, Njau S, Breen EC, Narr $\mathrm{KL}$, et al. Inflammation and improvement of depression following electroconvulsive therapy in treatment-resistant depression. J Clin Psychiatry 2018;79:17m11597.

23. Asoğlu M, Göbelek M, Karka İ, Pirinççioglu F, Çelik H, Ay $\mathrm{H}$, et al. Effect of electroconvulsive therapy on hematological 
parameters. Bezmialem Science 2018;6:130-3.

24. Fluitman SB, Heijnen CJ, Denys DA, Nolen WA, Balk FJ, Westenberg HG. Electroconvulsive therapy has acute immunological and neuroendocrine effects in patients with major depressive disorder. J Affect Disord 2011;131:388-92.

25. Chaturvedi S, Chadda RK, Rusia U, Jain N. Effect of electroconvulsive therapy on hematological parameters. Psychiatry Res 2001;104:265-8.

26. Khandaker GM, Cousins L, Deakin J, Lennox BR, Yolken R, Jones PB. Inflammation and immunity in schizophrenia: implications for pathophysiology and treatment. Lancet Psychiatry. 2015;2:258-70.

27. Patel PS, Buras ED, Balasubramanyam A. The role of the immune system in obesity and insulin resistance. J Obes 2013;2013:616193.

28. Rodríguez-Hernández H, Simental-Mendía LE, RodríguezRamírez G, Reyes Romero MA. Obesity and inflammation: epidemiology, risk factors andmarkers of inflammation. Int $\mathrm{J}$ Endocrinol 2013;2013:678159.

29. Balistreri CR, Caruso C, Candore G. The role of adipose tissue and adipokines in obesity-related inflammatory diseases. Mediators Inflamm 2010;2010:802078.

30. Fernandes B, Steiner J, Bernstein HG, Dodd S, Pasco JA, Dean OM, et al. C-reactive protein is increased in schizophrenia but is not altered by antipsychotics: meta-analysis and implications. Mol Psychiatry 2016;21:554-64.

31. Kweon OJ, Lee MK, Kim HJ, Chung JW, Choi SH, Kim HR. Neutropenia and neutrophil-tolymphocyte ratio in a healthy Korean population: race and sex should be considered. Int J Lab Hematol 2016;38:308-18.

32. Çakır U, Tuman TC, Yildırım O. Increased neutrophil/lymphoctye ratio in patients with bipolar disorder: a preliminary study. Psychiatr Danub 2015;27:180-4.

33. Kayhan F, Gündüz S, Ersoy SA, Kandeger A, Annagür BB. Relationships of neutrophil-lymphocyte andplatelet-lymphocyte ratios with the severity of major depression. Psychiatry Res 2017;247:332-5.

34. Mayda H, Ahsen A, Bagcioglu E, Öztürk A, Bahçeci B, Soyuçok E, et al. Effect of increased neutrophil-to-lymphocyte ratio (NLR) and decreased mean platelet volume (MPV) values on inflammation in acute mania. Arch Neuropsychiatry 2016;53:317-20.

35. Canan F, Kürklü N, Geçici Ö, Kuloglu M. The effect of electroconvulsive therapy on neutrophyl/lymphocyte ratio. JMOOD 2016;6:1-6.

36. Miller BJ, Buckley P, Seabolt W, Mellor A, Kirkpatrick B. Meta-analysis of cytokine alterations in schizophrenia: clinical status and antipsychotic effects. Biol Psychiatry 2011;70:663-71. 37. de Witte L, Tomasik J, Schwarz E, Guest PC, Rahmoune H, Kahn RS, et al. Cytokine alterations in first-episode schizophrenia patients before and after antipsychotic treatment. Schizophr Res 2014;154:23-9.

38. Wysokinski A, Szczepocka E. Platelet parameters (PLT, MPV,
P-LCR) in patients with schizophrenia, unipolar depression and bipolar disorder. Psychiatry Res 2016;237:238-45.

39. Lee J, Powell V, Remington G. Mean platelet volume in schizophrenia unaltered after 1 year of clozapine exposure. Schizophr Res 2014;157:134-6.

40. Ma J, Dou Y, Zhang H, Thijssen SW, Kuntsevich V, Ouellet $\mathrm{G}$, et al. Correlation between inflammatory biomarkers and red blood cell life span in chronic hemodialysis patients. Blood Purif 2017;43:200-5.

41. Ayyildiz H, Karabulut N, Kalayci M. Relationship between red blood cell distribution width and schizophrenia. Int J Med Biochem 2018;1:15-9.

42. Asoglu M, Aslan M, Canbolat O, Imre O, Savik E, Kivrak Y, et al. Red cell distribution width, mean platelet volume and vitamin B12 levels in patients with schizophrenia: an observational study. Int J Clin Exp Med 2016;9:16629-36.

43. Huang YL, Hu ZD. Lower mean corpuscular hemoglobin concentration is associated with poorer outcomes in intensive care unit admitted patients with acute myocardial infarction. Ann Transl Med 2016;4:190.

44. Balõtšev R, Haring L, Koido K, Leping V, Kriisa K, Zilmer $\mathrm{M}$, et al. Antipsychotic treatment is associated with inflammatory and metabolic biomarkers alterations among first-episode psychosis patients: A 7-month follow-up study. Early Interv Psychiatry 2019;13:101-9.

45. Maes M, Bocchio CL, Bignotti S, Battisa TG, Pioli R, Boin $\mathrm{F}$, et al. Effects of atypical antipsychotics on the inflammatory response system in schizophrenic patients resistant to treatment with typical neuroleptics. Eur Neuropsychopharmacol 2000;10:119-24.

46. Upthegrove R, Manzanares-Teson N, Barnes NM. Cytokine function in medication-naive first episode psychosis: a systematic review and meta-analysis. Schizophr Res 2014;155:101-8.

47. Semiz M, Yucel H, Kavakci O, Yildirim O, Zorlu A, Yilmaz $\mathrm{MB}$, et al. Atypical antipsychotic use is an independent predictor for the increased mean platelet volume in patients with schizophrenia: a preliminary study. J Res Med Sci 2013;18:5616.

48. Celebioglu B, Yigit H, Rezaki M, Ercelen O, Kayatekin S. Anesthesia in electroconvulsive therapy. Ann Saudi Med 1999;19:144-6.

49. Zhou R, Yang Z, Tang X, Tan Y, Wu X, Liu F. Propofol protects against focal cerebral 1schemia via inhibition of microglia-mediated proinflammatory cytokines in a rat model of experimental stroke. PLoS ONE 2013;8:e82729.

50. Samir A, Gandreti N, Madhere M, Khan A, Brown M, Loomba V. Anti-inflammatory effects of propofol during cardiopulmonary bypass: A pilot study. Ann Card Anaesth 2015;18:495-501.

51. Markovic-Bozic J, Karpe B, Potocnik I, Jerin A, Vranic A, Novak-Jankovic V. Effect of propofol and sevoflurane on the inflammatory response of patients undergoing craniotomy. BMC Anesthesiol 2016;16:18. 